\title{
Vorkommen und Diagnostik der OCD beim Hund
}

\author{
Susann Piesnack, Claudia Köhler, Vivian Fromme, Michaele Alef, Ingmar Kiefer
}

Die Osteochondrosis dissecans (OCD) ist bei Hunden eine häufig diagnostizierte Lahmheitsursache. Sie tritt in der Regel bei jungen, schnell wachsenden mittelgroßen und großen Hunden auf. Die Verdachtsdiagnose ergibt sich durch die Anamnese mit einer Krankheitshäufung im jugendlichen Alter ab dem 5. Monat, andererseits durch die Lahmheitsuntersuchung. Die Diagnosesicherung erfolgt dann durch die Bildgebung, meist durch die Röntgendiagnostik des entsprechenden Gelenks.

\section{Definition}

Die OCD ist eine erblich bedingte Störung der enchondralen Ossifikation. Sie manifestiert sich im Schulter-, Ellbogen-, Knie- und Sprunggelenk sowie am Kreuzbein unreifer mittelgroßer und großer Rassen. Das durch Retention degenerierte Knorpel- und subchondrale Knochengewebe bildet unter Verlust von Chrondrozyten einen Spalt an der Verbindungsstelle zwischen dem kalzifizierten und nicht kalzifizierten Gewebe. Schon durch eine normale Belastung können nun Risse entstehen, die die Loslösung einer Knorpelschuppe bewirken können $[3,9,16,25]$.

\section{Ätiologie}

Bei der OCD handelt es sich um eine multifaktorielle Erkrankung. Da in der Regel schnell wachsende mittelgroße und große Hunde betroffen sind und eine Häufung bei bestimmten Rassen beobachtet wird (beispielsweise Rhodesian Ridgeback, Boxer, Deutsche Dogge, Deutscher Schäferhund, Golden Retriever, Labrador Retriever), gehen verschiedene Autoren von einer genetischen Prädisposition aus [16, 18, 20, 25, 26]. Ein hoher Energiegehalt des Futters, unausgewogene Gehalte an Kalzium, Phosphat und
Vitamin D sowie hormonelle Imbalancen (Somatotropin, Schilddrüsenhormone, Andro- und Östrogene) sollen ebenso wie Traumata und Durchblutungsstörungen in den Epiphysenarterien einen Einfluss auf die Entstehung einer OCD haben [5, $10,24,27]$.

Rüden sind hierbei weitaus häufiger betroffen als Hündinnen. Obwohl die Hunde meist einseitige Lahmheiten zeigen, tritt die OCD häufig bilateral auf [9, 18].

\section{Pathogenese}

Bei der OCD handelt es sich im Wesentlichen um eine Störung der enchondralen Ossifikation des Gelenkknorpels. Aufgrund unterschiedlicher metabolischer Faktoren hypertrophieren die Knorpelzellen anstatt im Sinne der Chrondrogenese und Osteogenese zu degenerieren und kalzifizieren. Den Chondroklasten fehlt infolgedessen das Signal zur Mineralisation und sie bleiben inaktiv. Das resultiert in einer kontinuierlichen Verdickung des Gelenkknorpels. Dieses Stadium der Osteochondrose kann spontan abheilen oder sich zur OCD weiterentwickeln [22,24]. Ab einer kritischen Schichtdicke wird der Gelenkknorpel nur noch ungenügend mit synovialen Nährstoffen versorgt und stirbt ab. Mechanische Belastungen der betroffenen Bereiche führen zur Auflockerungen der Knochen-Knorpelgrenze, die sich zu Fissuren und schließlich zur Ablösung einer Knorpelschuppe weiterentwickeln kann [10, 22]. Erst jetzt spricht man von einer OCD. Häufig bleibt diese Knorpelschuppe (Dissekat) über einen dünnen medial gelegenen Steg mit dem umliegenden Gelenkknorpel verbunden. Dies wird dann als Knorpelflap bezeichnet. Löst sie sich aber vollständig von der Knorpelschicht, bildet sie einen freien Gelenkkörper (Corpus liberum oder Gelenkmaus). Heften sich Corpora libera an die Gelenkkapsel an, werden sie durch die Synovia ernährt, können weiter wachsen und schließlich auch verknöchern [26]. Sowohl Knorpelschuppe als auch Corpus liberum können zusätzlich eine Sehnenscheidenentzündung verursachen, die nach verschieden langer Zeit in der Regel eine Arthropathia deformans (sekundäre Arthrose) verursacht $[18,22,24]$.

\section{Lokalisationen und Alter}

Die häufigsten Lokalisationen der OCD beim Hund sind der zentrokaudale Anteil des Caput humeri, die Trochlea humeri, der Condylus lateralis ossis femoris sowie die Trochlea tali medialis ( $\vee$ Abb. 1) $[7,14,16]$. Hierbei liegt das typische Alter für die klinisch apparente Erkrankung im Schultergelenk bei 5-7 Monaten. Im Ellbogengelenk erkranken die Hunde durchschnittlich mit 4-5 Monaten. Für das Kniegelenk liegt das Erkrankungsalter im Durchschnitt bei 6-8 Monaten, im Tarsalgelenk bei 4-6 Monaten [24]. Weiterhin können vereinzelt auch der zentrale Anteil der Cavitas glenoidalis scapulae, der Condylus medialis ossis femoris sowie die Trochlea tali lateralis betroffen sein $[8,13,18,19,21]$. Bei der vertebralen beziehungsweise sakralen OCD sind die kaudale Endplatte des L7 und/oder die kraniale Endplatte des Sakrums betroffen $[17,19]$.

Typischerweise wird die Erkrankung durchschnittlich 1-4 Monate nach dem vermutlichen Auftreten der OCD diagnostiziert. Aufgrund des akuten Auftretens und der guten Erkennbarkeit der Lahmheit wird die OCD im Ellbogengelenk besonders früh diagnostiziert. Im Schultergelenk erfolgt die röntgenologische Diagnosesicherung durch die verzögerte Ausbildung einer sekundären 


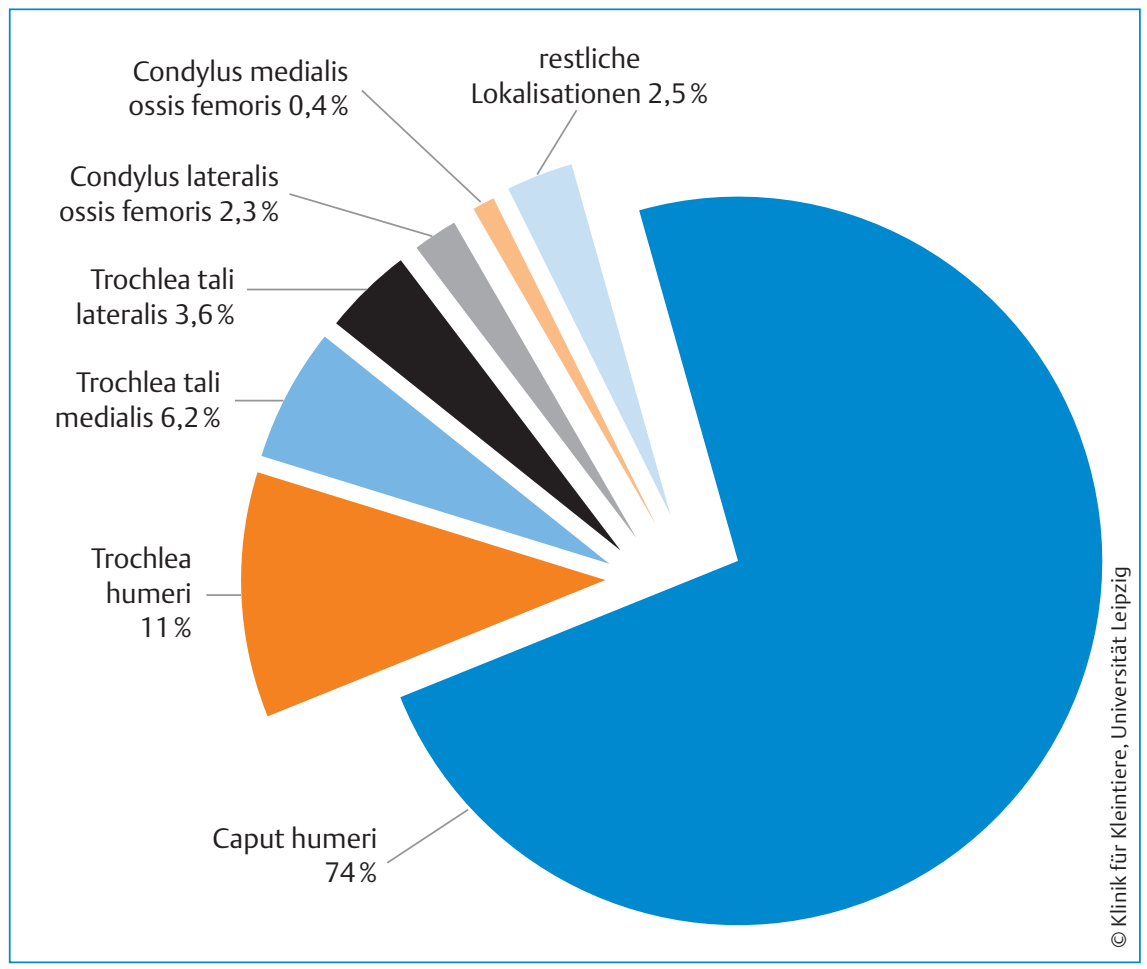

Abb. 1 Lokalisationen und Häufigkeit der OCD beim Hund [14, 16, 20].

Arthrose zeitlich meist etwas später, gefolgt von der OCD im Tarsokruralgelenk und Kniegelenk [16, 25].

\section{Klinische Untersuchung}

Obwohl die Hunde meist bilateral erkranken, werden sie zunächst mit einer einseitigen Lahmheit vorgestellt. Typi-

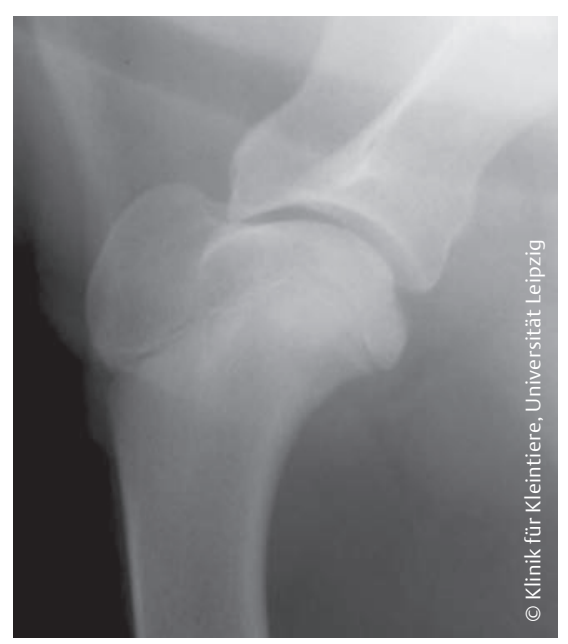

Abb. 2 OCD des Schultergelenks. Ein unregelmäßiger Defekt im kaudalen Bereich des Humeruskopfs ist erkennbar. Auf den Nativaufnahmen ist die nicht mineralisierte Knorpelschuppe zu erkennen. scherweise wird die Erkrankung 1-4 Monate nach dem Auftreten diagnostiziert. Die Lahmheit kann unterschiedlichen Grades vorliegen, meist mit einer deutlichen Schrittverkürzung. Nach Ruhe, aber auch nach Belastung wird diese Lahmheit beobachtet. Aber besonders bei beidseitiger Erkrankung kann auch nur eine Steifheit nach dem Liegen er-

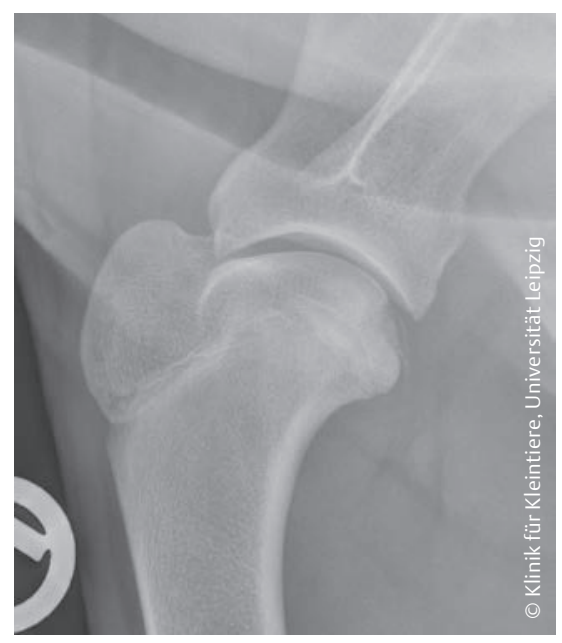

Abb. 3 OCD des Schultergelenks. Es stellt sich ein unregelmäßiger Defekt im kaudalen Bereich des Humeruskopfs mit mineralisierter Knorpelschuppe dar. kennbar sein. Bei der klinischen Untersuchung liegt in der Regel eine vermehrte Gelenkfüllung vor. Die passive Beugung und Streckung des Gelenks ist meist schmerzhaft [27, 28].

\section{Diagnostik}

Die Diagnosesicherung erfolgt durch konventionelle Röntgenaufnahmen des entsprechenden Gelenks.

\section{Aufgrund der hohen Inzidenz bilateraler Läsionen wird empfohlen, immer beide Gliedmaßen zu röntgen.}

Sollten hierbei unklare Befunde vorliegen, so ist meist durch die CT-Diagnostik eine endgültige Klärung möglich. Die magnetresonanztomografische Diagnostik der OCD erlaubt eine detaillierte Darstellung von Gelenkknorpel, Synovia und Knochenmark, ist aber nur in Ausnahmefällen wirklich erforderlich. Selbst noch nicht kalzifizierte Fragmente können mit hoher Sicherheit identifiziert werden. Weiterhin können Anzeichen akuter Entzündungen in Gelenken mit noch intaktem Gelenkknorpel dargestellt werden [27, 28].

\section{Projektionsradiografie Schultergelenk}

Auch wenn die Lahmheit an nur einer Gliedmaße offensichtlich ist, sollten stets beide Schultergelenke radiologisch untersucht werden $[3,26]$. Der häufigste Röntgenbefund bei der OCD der Schulter besteht in einer Abflachung im kaudalen Anteil des Humeruskopfs. Weiterhin ist ein unregelmäßiger, strahlendurchlässiger, subchondraler Defekt an der Kaudalfläche des Caput humeri möglich ( Abb. 2). Liegen kalzifizierte Corpora libera vor, so befinden sich diese für gewöhnlich über dem subchondralen Defekt ( $\triangleright$ Abb.3). Als zusätzlicher Röntgenbefund kann ein sklerotischer Randsaum im Bereich des Defekts erhoben werden. Im weiteren Verlauf kommt es sekundär zu einer Osteoarthrose. Zur Beurteilung des Zustands des Gelenkknorpels oder zur Darstellung nicht mineralisierter Knorpelschuppen eignen sich Nativaufnahmen jedoch nicht. Hier kann die Arthrografie mit Positivkontrastmittel manchmal hilfreich sein, wenngleich 


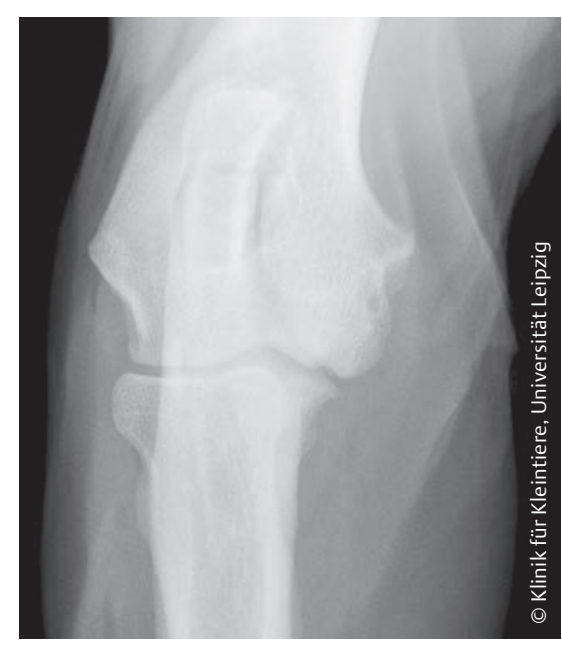

Abb. 4 OCD des Ellbogengelenks. Auf der kraniokaudalen Röntgenaufnahme ist ein subchondraler Defekt an der distalen Kontur der medialen Humeruskondyle erkennbar.

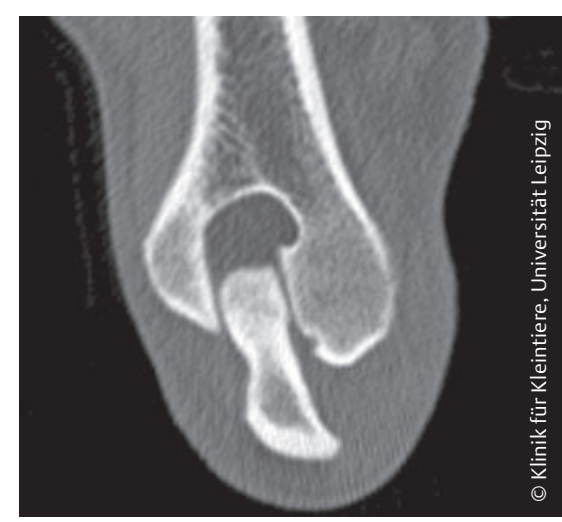

Abb. 5 CT-Bild eines Hundes mit OCD: Defekt (Abflachung) des subchondralen Knochens medial an der Humeruskondyle.

die MRT-Diagnostik bei der Beurteilung des Knorpels die Methode der Wahl darstellt.

\section{Projektionsradiografie Ellbogengelenk}

Zur röntgenologischen Darstellung von OCD-Läsionen im Bereich des Ellbogengelenks werden standardmäßig 4 Projektionen angefertigt [10, 14, 15, 16, 24, 31]:

- mediolateral neutral

- mediolateral gebeugt

- kraniokaudal

- kraniolateral-kaudomediale Schrägaufnahmen (15-Projektion)

In der kraniokaudalen oder der schrägen Aufnahme fällt zunächst eine konkave beziehungsweise halbmondförmige Aufhellung des distalen medialen Humeruskondylus auf. Im späteren Stadium wird ein ausgedehnter Defekt mit subchondraler Sklerose sichtbar ( $\bullet$ Abb.4). Des Weiteren kann eine Abflachung der kranioventralen Fläche der Trochlea humeri dargestellt werden. Eine isolierte Schuppe, entsprechend der OCD im Schultergelenk, ist fast nie zu erkennen $[15,18$, 22]. Sollte die OCD in der Projektionsradiografie nicht eindeutig zu diagnostizieren sein, sind weitere Untersuchungsverfahren wie die Computertomografie ( Abb.5), die Magnetresonanztomografie oder die Arthroskopie zur Sicherung der Diagnose heranzuziehen [18].

\section{Projektionsradiografie Kniegelenk}

Für die Darstellung der OCD am lateralen und medialen Condylus femoris wird eine mediolaterale und eine kraniokaudale oder kaudokraniale Röntgenaufnahme benötigt. Obwohl die mediolaterale Aufnahme häufig schon pathognomonische Anzeichen der OCD zeigt, ist eine kraniokaudale oder kaudokraniale Projektion erforderlich, um festzustellen, welcher Kondylus betroffen ist [4].

Die Röntgenbilder können Veränderungen wie eine Abflachung der Gelenk-

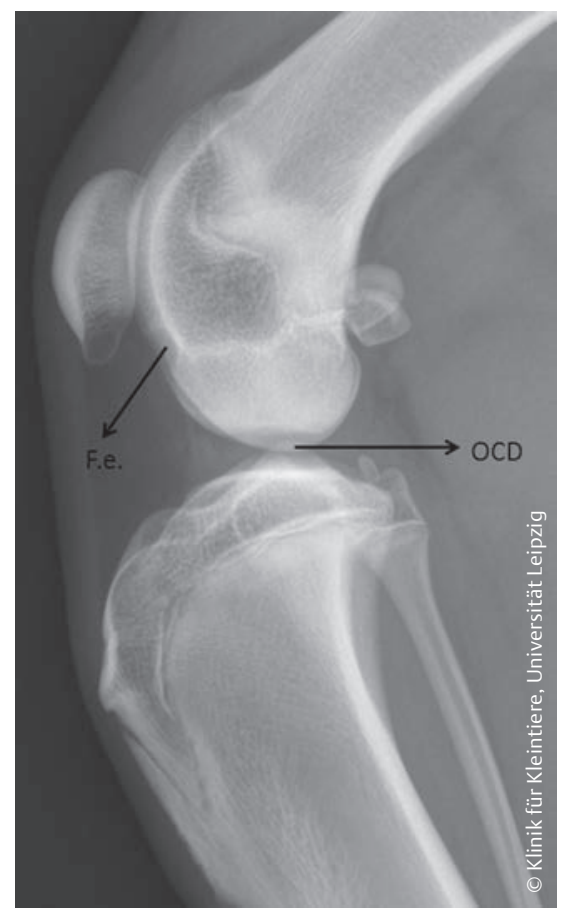

Abb. 6 OCD des Kniegelenks. Auf der mediolateralen Aufnahme ist ein strahlentransparenteres Areal im Femurkondylus im Sinne von knöchernem Substanzverlust zu erkennen. Eine Seitenzuordnung ist hier nicht möglich. Zusätzlich Illustration zur Unterscheidung der Fossa extensoria (F.e.) und der OCD-Läsion. oberfläche an einem der beiden Femurkondylen (meist Medialfläche lateraler Kondylus), einen konkaven Defekt am subchondralen Knochen (mit oder ohne angrenzende Sklerose) oder selten mineralisierte Knorpelschuppen zeigen ( Abb.6, \Abb. 7).

Differenzialdiagnostisch kann es zu einer Verwechslung der normalen Fossa extensoria (Ursprung M. extensor digitorum longus) mit einer OCD-Läsion kommen. Die Fossa resultiert aber im Gegensatz zur OCD-Läsion nicht in einem Defekt der Gelenkfläche (kraniokaudale Projektion) und ist auf der mediolateralen Aufnahme deutlich weiter kranial und proximal lokalisiert ( Abb. 6) [4, 22]. Zur besseren Diagnosesicherung, vor allem bei Verdacht auf kleinere Läsionen im Bereich des Kniegelenks, bietet sich die Computertomografie an ( $\triangleright$ Abb. 7 , -Abb. 8) [18].

\section{Projektionsradiografie Tarsokruralgelenk}

Standardmäßig werden zur Projektion des Tarsalgelenks eine mediolaterale und eine dorsoplantare/plantodorsale Auf-

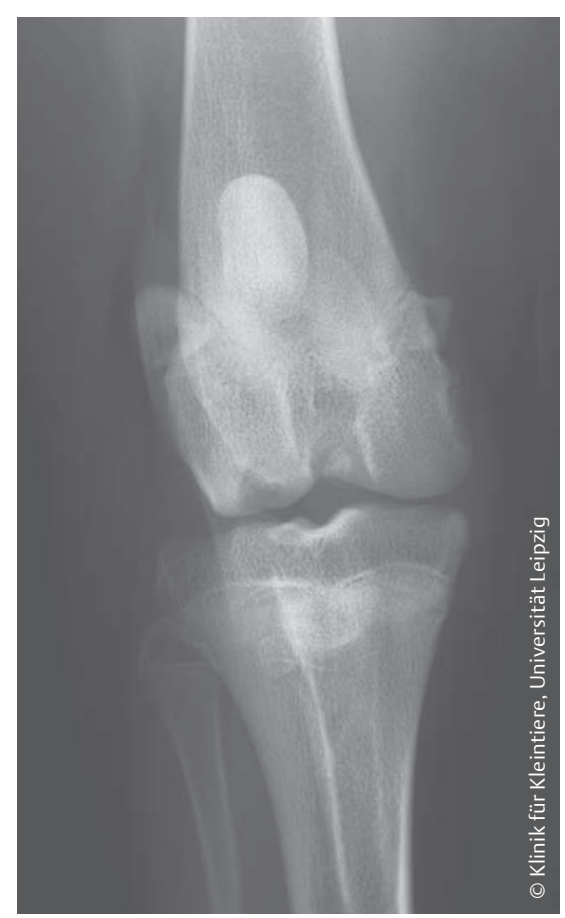

Abb. 7 OCD des Kniegelenks. In der kaudokranialen Aufnahme ist ein subchondraler Defekt distal am lateralen Femurkondylus mit angrenzendem sklerotischem Saum zu erkennen. Im Zentrum befindet sich ein mineralisiertes Fragment. 
nahme angefertigt. Besteht der klinische Verdacht einer OCD und lassen sich auf den genannten Projektionen aber keine Hinweise dafür feststellen, werden dorsoplantare Aufnahmen mit Flexion des Tarsus angeraten (Skyline) [1, 22].

Häufig ist eine Abflachung des medialen Talusrollkamms im Sinne eines subchondralen Knochendefekts zu erkennen; seltener auch des lateralen Rollkamms. Im Bereich des Defekts ist im Falle einer Mineralisation eine isolierte Knorpelschuppe zu erkennen. Die schnell einsetzende, meist hochgradige Arthrose führt zusätzlich zu einer unruhigen proximalen Kontur ( $\triangleright$ Abb.9). Ein weiterer Hinweis ist ein erweiterter medialer Gelenkspalt des Tarsokruralgelenks [1, 18, 22]. Mithilfe der Computertomografie können Ausmaß und Lokalisation der Defekte jedoch weitaus sicherer dargestellt werden [11].

\section{Projektionsradiografie OCD Kreuzbein}

Verglichen mit der Diagnostik der OCD in Ellbogen oder Sprunggelenk ist die Diagnosestellung bei einer OCD des Kreuzbeins vergleichsweise einfach. In der Regel ist eine korrekt gelagerte laterolaterale Aufnahme ausreichend. Die vertebrale beziehungsweise sakrale OCD ist durch eine sklerotische Veränderung im dorsalen Bereich der knorpeligen Endplatten von L7 (kaudale Endplatte) beziehungsweise des Sakrums (kraniale Endplatte) gekennzeichnet. Es können ein oder mehrere Fragmente im Wirbelkanal zu liegen kommen. Diese bestehen aus hyalinem Knorpel und besitzen ein knöchernes Zentrum. Bedingt durch die OCD kann es zu degenerativen Veränderun- gen der Bandscheibe kommen. Das Cauda-Equina-Syndrom tritt dann klinisch zu einem früheren Zeitpunkt als gewöhnlich in Erscheinung. Schäferhunde zeigen für diese Art der OCD eine besondere Prädisposition [12, 17, 19].

\section{Therapie}

Mit strikter Ruhighaltung und Futterumstellung kann bei Junghunden (<6 Monate), vor allem, wenn röntgenologische Veränderungen fehlen, ein konservativer Therapieversuch erfolgen. Dieser Therapieansatz wird aber durchaus als kritisch angesehen. Ebenfalls ist eine solche Versorgung bei älteren Hunden mit länger bestehender OCD im Knieoder Tarsalgelenk und minimaler Lahmheitssymptomatik möglich [9], aber auch nicht unumstritten.

Bei persistierender Lahmheit wird die operative Entfernung der Knorpelschuppe und eine vorsichtige Kürettage des darunterliegenden beeinträchtigten Knochens empfohlen. Auf diesem Wege heilt der Defekt ab und Faserknorpel kann gebildet werden. Dieser Eingriff kann durch eine Arthrotomie oder Arthroskopie erfolgen. Der Patient sollte nach der Operation für etwa 6 Wochen mit strengem Leinenzwang ruhig gehalten werden [8]. Danach kann eine leichte Steigerung der Bewegung erfolgen. Begleitend wird zum Aufbau der Muskulatur Physiotherapie empfohlen $[9,18]$.

\section{Prognose}

An OCD leidende Hunde, die konservativ versorgt werden, zeigen in vielen Fällen weiterhin intermittierende Lahmheiten

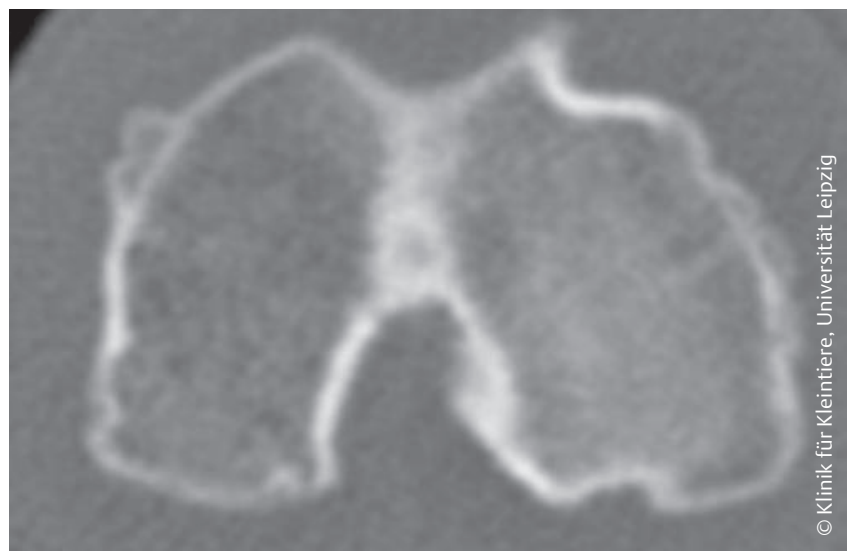

Abb. 8

CT-Bild eines Hundes mit OCD. Substanzverlust im Bereich des lateralen Femurkondylus mit angrenzendem sklerotischem Saum. Weiterhin hochgradige osteophytäre Zubildungen.
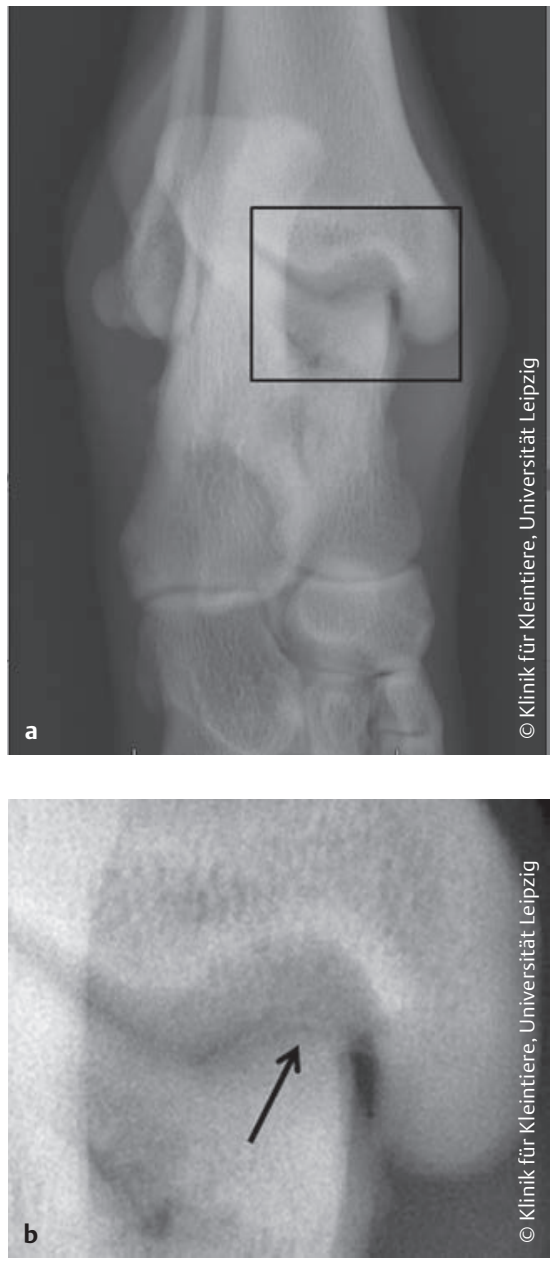

Abb. 9a und b Tarsus eines Hundes mit OCD. Die intrakapsuläre Weichteilschwellung weist auf einen Gelenkerguss hin. Weiterhin sind eine osteochondrale Fragmentation des medialen Talusrollkamms (Pfeil) und osteophytäre Zubildungen im Sinne einer Arthrose zu erkennen.

und fortschreitende degenerative Veränderungen des entsprechenden Gelenks [9]. Nach einem chirurgischen Eingriff ist die Prognose der OCD im Schultergelenk hinsichtlich einer normalen Schultergelenkfunktion günstig. Jedoch kann die Arthrose trotz der Behandlung fortschreiten. Diese geht dann aber nur selten mit einer erneuten Lahmheit einher [18].

Obwohl Hunde mit einer OCD im Ellbogen-, Knie- und Tarsalgelenk post OP eine verbesserte Gliedmaßenfunktion aufweisen, ist die Prognose in Bezug auf eine normale Gelenkfunktion vorsichtig zu beurteilen. Die fortschreitende Degeneration dieser Gelenke und der irreversible Knorpelverlust erfordern häufig eine medikamentöse Behandlung [6,9, 
29]. Auch wenn nach der Operation eine deutliche Besserung eintritt, so sollte der Einsatz als Gebrauchshund oder Sporthund sehr kritisch überdacht werden.

\section{Schlussfolgerung}

Die OCD ist eine Erscheinungsform der Osteochondrosis, die häufig bilateral auftritt. Operativ behandelte Hunde zeigen für gewöhnlich eine Verbesserung der Gliedmaßenfunktion. Jedoch sollte der Besitzer darüber aufgeklärt werden, dass die meisten betroffenen Tiere trotz des Eingriffs fortschreitende degenerative Gelenkveränderungen zeigen, die unter Umständen eine medikamentöse Behandlung erfordern. Da die OCD eine erbliche Komponente hat, sollten die Besitzer zusätzlich davor gewarnt werden, mit den betroffenen Hunden zu züchten [9]. In den meisten Verbänden führt eine OCD ohnehin zum Zuchtausschluss.

\section{Online zu finden unter}

http://dx.doi.org/10.1055/s-0042-120601

\section{Literatur}

1 Allan G, Nicoll R. Distaler Abschnitt der Gliedmaßen - Karpus und Tarsus. In: Barr F], Kirberger RM, Hrsg. Bildgebende Diagnostik des muskuloskelettalen Systems bei Hund und Katze. München: Elsevier; 2009: 187-216

2 Boudrieau RJ, Hohn RB, Bardet JF. Osteochondritis dissecans of the elbow in the dog. J Am Anim Hosp Assoc 1983; 19: 627-635

3 Brunnberg L, Waibl H, Nagel ML. Zur aseptischen Knochennekrose des Caput humeri beim Hund. Berl Munch Tierarztl Wochenschr 1978; 91: 418-423

4 Carlson CS, Cullins LD, Meuten DJ. Osteochondrosis of the articular-epiphyseal cartilage complex in young horses: evidence for a defect in cartilage canal blood supply. Vet Pathol 1995; 32: 641

5 Comerford Ej. Kniegelenk. In: Barr F], Kirberger RM, Hrsg. Bildgebende Diagnostik des muskuloskelettalen Systems bei Hund und Katze. München: Elsevier; 2009: 181-182

6 Coppieters E, Gielen E, Verhoeven G et al. Erosion of the medial compartment of the canine elbow: occurence, diagnosis and currently available treatment options. Vet Comp Orthop Traumatol 2015; 28: 9-18
7 Fayolle P, Ormieres P, Autefage A et al. Osteochondrose du grasset chez le chien. Synthese bibliographique et presentation d'un cas. Prat méd Chir Anim Comp 1987; 22 : 41-53

8 Fitch RB, Beale BS. Osteochondrosis of the canine tibiotarsal joint. Vet Clin North Am Small Anim Pract 1998; 28: 95-113

9 Fossum TW. Gelenkerkrankungen. In: Fossum TW, Hrsg. Chirurgie der Kleintiere. München, Jena: Urban \& Fischer/Elsevier; 2006: 1123-1668

10 Fox SM, Walker AM. The etiopathogenesis of osteochondrosis. Vet Med 1993; 88: 116-118

11 Gielen I, van Bree H, Van Ryssen B et al. Radiographic, computed tomographic and arthroscopic findings in 23 dogs with osteochondrosis of the tarsocrural joint. Vet Rec 2002; 150: 442-447

12 Hanna FY. Lumbosacral osteochondrosis: radiological features and surgical management in 34 dogs. J Small Anim Pract 2001; 42: 272-278

13 Harari J. Osteochondrosis of the femur. Vet Clin North Am 1998; 28: 87-94

14 Kippenes H, Johnston G. Diagnostic imaging of osteochondrosis. Vet Clin North Am Small Anim Pract 1998; 28: 137-160

15 Kirberger RM. Ellenbogengelenk. In: Barr F], Kirberger RM, Hrsg. Bildgebende Diagnostik des muskuloskelettalen Systems bei Hund und Katze. München: Elsevier; 2009: 135-145

16 Krone O. Osteochondrosis dissecans beim Hund: Darstellung von Defekten im Schulter-, Ellbogen-, Knie- und Sprunggelenk sowie am Kreuzbein mittels Computertomographie [Dissertation]. Hannover: Tierärztliche Hochschule Hannover; 2002

17 Lang J, Häni H, Schawalder P. A sacral lesion resembling osteochondrosis in the German Shepherd Dog. Vet Radiol 1992; 33: 69-77

18 Meyer-Lindenberg A, Reif $\mathrm{U}$, Wagner $\mathrm{F}$. Spezielle Erkrankungen im Bereich der Gelenke, Muskeln und Sehnen. In: Suter BF, Kohn B, Schwarz G, Hrsg. Praktikum Hundeklinik. Stuttgart: Enke; 2012: 998-1071

19 Milton JL, Rumph PF, Reed AD. Osteochondritis dissecans of the shoulder in the racing Greyhound: a report of two cases and a survey of 109 Greyhound anatomy specimens. J Am Anim Hosp Assoc 1981; 17 : 617-622

20 Montgomery RD, Milton JL, Hathcock JT et al. Osteochondritis dissecans of the canine tarsal joint. Compend Contin Educ Pract Vet 1994; 16: 835-845

21 Montgomery RD, Milton JL, Henderson RA et al. Osteochondritis dissecans of the canine stifle. Compend Contin Educ Pract Vet 1989; 11: 1199-1205
22 Morandi F, Hecht S, Daniel GB. Knochen- und Gelenkerkrankungen bei Jungtieren. In: Hecht S, Hrsg. Röntgendiagnostik in der Kleintierpraxis. Stuttgart: Schattauer; 2008: 343-360

23 Olsson SE. Pathophysiology, morphology, and clinical signs of osteochondrosis (chondrosis) in the dog. In: Bojrab MN, ed. Disease Mechanisms in Small Animal Surgery. Philadelphia, Pennsylvania, USA: Lea and Febiger; 1993: 777-789

24 Schebitz H, Wilkens H. Atlas der Röntgenanatomie von Hund und Katze, Hrsg. Berlin, Hamburg: Paul Parey; 1989: 56-102

25 Slater MR, Scarlett JM, Kaderly RE et al. Breed, gender, and age as risc factors for canine osteochondrosis dissecans. Vet Comp Orthop Traumatol 1991; 4: 100-106

26 van Bree H, Gielen I. Schultergelenk und Scapula. In: Barr F], Kirberger RM, Hrsg. Bildgebende Diagnostik des muskuloskelettalen Systems bei Hund und Katze. München: Elsevier; 2009: 114-121

27 van Bree H, van Ryssen B, Degryse H et al. Magnetic resonance arthrography of the scapulohumeral joint in dogs, using gadopentetate dimeglumine. Am J Vet Res 1995; 56: 286-288

28 van Bree H, Degryse H, van Ryssen B et al. Pathologic correlations with magnetic resonance images of osteochondrosis lesions in canine shoulders. J Am Vet Med Assoc 1993; 202: 1099-2005

29 Van der Peijl G], Schaeffer IG, Theyse LF et al. Osteochondrosis dissecans of the tarsus in Labrador Retrievers: clinical signs, radiological data and force plate gait evaluation after surgical treatment. Vet Comp Orthop Traumatol 2012; 25: 126-134

30 Weinstein MJ, Mongil CM, Smith GK. Orthopedic conditions of the rottweiler. Comp Cont Ed 1995; 17: 813-838

31 Zentek J, Nolte I. Osteochondrosen bei einem neunmonatigen Berner Sennenhund. Kleintier Prax 1995; 40: 879-887

Dr. Susann Piesnack
Dr. Claudia Köhler
TÄ Vivian Fromme
Prof. Dr. Michaele Alef, Dipl ECVAA,
FTÄ für Chirurgie und Anästhesiologie
Dr. Ingmar Kiefer
Universität Leipzig
Klinik für Kleintiere
An den Tierkliniken 23
04103 Leipzig
Kiefer@kleintierklinik.uni-leipzig.de




\section{Fragebogen}

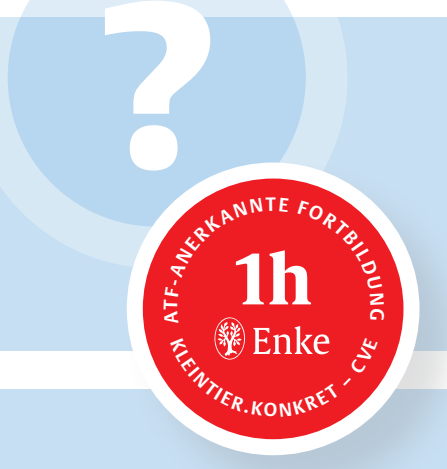

\section{Frage 1}

Die OCD hat in Bezug auf das betroffene Gelenk global eine unterschiedliche Prognose auf die funktionelle Wiederherstellung. Welches Gelenk besitzt die beste Prognose?
a. Sprunggelenk
b. Kniegelenk
c. Ellbogengelenk
d. Schultergelenk
e. Os sacrum

\section{Frage 2}

Einzelne Rassen haben für bestimmte Formen der OCD eine Disposition. Bei welcher Rasse trifft dies für die OCD im Bereich des Os sacrum zu?
a. Golden Retriever
b. Labrador Retriever
c. Dogge
d. Deutscher Schäferhund
e. Rottweiler

\section{Frage 3}

In welchem Gelenk kann am ehesten ein Dissekat in der Projektionsradiografie nachgewiesen werden?
a. Schulter
b. Ellbogen
c. Knie
d. Sprunggelenk
e. Os sacrum

\section{Frage 4}

Ab welchem Alter tritt die OCD im Ellbogengelenk typischerweise klinisch auf?
a. 4 Monate
b. 7 Monate
c. 10 Monate
d. 13 Monate
e. 15 Monate

\section{Frage 5}

An welchem Gelenk wird die OCD beim Hund am häufigsten diagnostiziert?
a. L7/Os sacrum
b. Tarsalgelenk
c. Karpalgelenk
d. Ellbogengelenk
e. Schultergelenk

\section{Frage 6}

Wie viele Aufnahmen werden bei der standardmäßigen röntgenologischen OCD-Diagnostik eines Ellbogengelenks angefertigt?
a. 2
b. 3
c. 4
d. 5
e. 6

\section{Frage 7}

Welcher Bereich ist bei der OCD des Knies relativ häufig betroffen?
a. kaudales Tibiaplateau
b. medial am lateralen Kondylus
c. lateral am lateralen Kondylus
d. medial am medialen Kondylus
e. lateral am medialen Kondylus

\section{Frage 8}

Bei der Diagnostik der OCD wird mintunter auch eine Skyline-Aufnahme angefertigt. Bei welchem Gelenk profitiert der Untersucher von dieser Methode am meisten?
a. Schultergelenk
b. Ellbogengelenk
c. Tarsalgelenk
d. Kniegelenk
e. Karpalgelenk

Frage 9

Mit welchem Prozentsatz ist die Trochlea tali medialis an der Gesamtverteilung der OCD-Erkrankungen beim Hund ungefähr beteiligt?
a. $2 \%$
b. $4 \%$
c. $6 \%$
d. $8 \%$
e. $11 \%$

\section{Frage 10}

Welcher anatomische Anteil des Sprunggelenks ist neben der Trochlea tali medialis vergleichsweise häufig an der Erkrankung beteiligt?
a. Trochlea tali lateralis
b. Calcaneus
c. Os tarsale 3
d. Os tarsale 4
e. Os metatarsale 3 


\section{Vorkommen und Diagnostik der OCD beim Hund}

Susann Piesnack, Claudia Köhler, Vivian Fromme, Michaele Alef, Ingmar Kiefer

A Lernerfolgskontrolle

\section{Bitte kreuzen Sie die richtigen Antworten an! Es ist jeweils nur 1 Antwort pro Frage richtig!}

\section{B Teilnehmer}

\begin{tabular}{llll|l|l|l|l|l|l|l|l|l|l|} 
Frage 1 & a & b & c & d & e & & Frage 6 & a & b & c & d & e \\
Frage 2 & a & b & c & d & e & Frage 7 & a & b & c & d & e \\
Frage 3 & a & b & c & d & e & Frage 8 & a & b & c & d & e \\
Frage 4 & a & b & c & d & e & Frage 9 & a & b & c & d & e \\
Frage 5 & a & b & c & d & e & Frage 10 & a & b & c & d & e \\
\hline
\end{tabular}

\section{E Erklärung}

Ich versichere, dass ich die Beantwortung der Fragen selbst und ohne Hilfe durchgeführt habe.

\section{Ihr Ergebnis wird vom Verlag ausgefültt}

Sie haben von $\quad \square$ bestanden und 1 ATF-Stunde erhalten.

Stuttgart, den

D Teilnahmebedingungen für Abonnenten der kleintier konkret kostenlos

Für diese Fortbildungseinheit können Sie 1 ATFFortbildungsstunde anerkannt bekommen. Hierfür

müssen mindestens $70 \%$ der Fragen richtig beantwortet sein.

muss der Antwortbogen vollständig ausgefültt sein. Unvollständig ausgefüllte Bögen können nicht berücksichtigt werden! muss im markierten Feld* Ihre Abonnentennummer eingetragen oder eine kleintier. konkret-CVE-Wertmarke aufgeklebt sein.

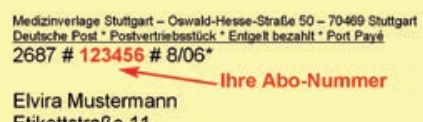

Stempel | Unterschrift

kleintier.konkret-CVE-Wertmarken für NichtAbonnenten können beim Verlag zu folgenden Bedingungen erworben werden: 6er-PackWertmarken, Preis 49,95€ inkl. MWSt., Artikel-Nr. 903000.

Bitte richten Sie die Bestellungen an: Georg Thieme Verlag Stuttgart,

KundenServiceCenter Buch, Postfach 3011 20, 70451 Stuttgart.

* Nicht-Abonnenten bitte hier kleintier. konkret-CVE-Wertmarke aufkleben, Abonnenten bitte Abonnentennummer eintragen. 